This PDF is a selection from a published volume from the National Bureau of Economic Research

Volume Title: Insights in the Economics of Aging

Volume Author/Editor: David A. Wise, editor

Volume Publisher: University of Chicago Press

Volume ISBNs: 0-226-42667-X; 978-0-226-42667-9 (cloth);

978-0-226-42670-9 (e-ISBN)

Volume URL: http://www.nber.org/books/wise-21

Conference Date: April 30-May 2, 2015

Publication Date: March 2017

Chapter Title: Are Black-White Mortality Rates Converging? Acute Myocardial Infarction in the United States, 1993-2010

Chapter Author(s): Amitabh Chandra, Tyler Hoppenfeld, Jonathan Skinner Chapter URL: http://www.nber.org/chapters/c13643

Chapter pages in book: (p. $205-222$ ) 


\title{
Are Black-White Mortality Rates Converging? Acute Myocardial Infarction in the United States, 1993-2010
}

\author{
Amitabh Chandra, Tyler Hoppenfeld, and \\ Jonathan Skinner
}

\subsection{Introduction}

There is a vast literature documenting the presence of pervasive racial disparities in US health care (IOM 2002). More recently, researchers have studied changes over time in the extent of racial and socioeconomic disparities, to test whether the public focus on disparities in health care has led to fundamental changes in practice styles, improved sensitivity by health care providers to different cultural norms, and less biased treatment and outcome decisions. In many cases, there has been a notable reduction in the magnitude of disparities in treatment and the use of "effective" care (Trivedi et al. 2005; Trivedi et al. 2011; Jha et al. 2005). There is less progress, however, with respect to racial disparities in overall health outcomes (e.g., Meara, Richards, and Cutler 2008).

How can this puzzle be explained? Many efforts to address disparities have focused on how physicians treat patients of different races and ethnicities. For example, the public knowledge that stenting rates for black patients are so much lower than those for white patients could lead to cardiologists at the margin to question their decisions not to provide stents to their black patients. An increasing share of minority health professionals could also

Amitabh Chandra is the Malcolm Wiener Professor of Social Policy and Director of Health Policy Research at the Harvard Kennedy School of Government and a research associate of the National Bureau of Economic Research. Tyler Hoppenfeld is a research fellow in the Evidence for Policy Design program at Harvard University. Jonathan Skinner is the James O. Freedman Presidential Professor of Economics at Dartmouth College, professor of community and family medicine at the Dartmouth Institute for Health Policy and Clinical Practice, and a research associate of the National Bureau of Economic Research.

For acknowledgments, sources of research support, and disclosure of the authors' material financial relationships, if any, please see http://www.nber.org/chapters/c13643.ack. 
lead to a decline in the extent of disparities in treatments of diverse patient populations. Over a sufficiently lengthy period of time, we might expect to see a convergence in treatment patterns as a result of efforts to reduce both implicit and explicit biases in health care within the hospital.

A much different source of health disparities arises from the fact that black and white patients go to different providers. One study, for example, documented that nearly half of all black acute myocardial infarction (AMI) patients were admitted to 571 hospitals serving just 7 percent of white AMI patients (Skinner et al. 2005). The authors estimated that most of the gap between black and white ninety-day mortality was the consequence of the quality of the hospital to which the patient was admitted, and not because of how black and white patients were treated within the hospital.

Should we expect a convergence in this quality differential across hospitals? Unfortunately, even if all physicians and health professionals were entirely bias-free with regard to race and ethnicity, we would still observe disparities in outcomes if black or Hispanic patients were admitted to hospitals with lower average quality. The question of convergence here is related to two quite different factors: whether patient demand leads to migration toward high-quality hospitals (Chandra et al. 2013), and whether there is a convergence in the quality of hospitals as poor-performing hospitals catch up over time (Skinner and Staiger 2015).

To test these hypotheses, this chapter uses a two-decade time-series approach to test for convergence in hospital quality between black and white patients admitted to hospital for acute myocardial infarction, or AMI. Using a sample of more than four million patients, we do not find evidence of convergence of black and white ninety-day mortality rates. From 1993 to 1998, black AMI patients experienced risk-adjusted mortality rates 0.4 percentage points greater than white AMI patients. After increasing to a gap of 1.6 percentage points from 1999 to 2005, by 2006-2010 the gap had been attenuated to 1.0 percentage points, still more than double the initial disparity in 1993-1998.

With regard to the sources of this widening in outcomes, we use an Oaxaca-Blinder decomposition to consider separately the "within-hospital" sources of racial disparities in outcomes and the "between-hospital" measure capturing black AMI patients going to hospitals with higher (white) mortality rates. Of the overall racial disparity in risk-adjusted outcomes, most was the consequence of between-hospital differences in quality; black patients were admitted to lower-quality hospitals (where quality was measured by risk-adjusted outcomes for white patients), and these betweenhospital disparities have shown little evidence of convergence.

At the same time, the within-hospital disparities have been growing. One potential explanation for such differences is the use of percutaneous coronary interventions (PCI), which includes angioplasty and stenting, during the index admission - a marker for "primary" and highly effective surgical 
treatment for AMI. We find indeed that while rates of PCI trended upward for both black and white patients, the trend was more pronounced for white patients within hospitals - in other words, the rapid diffusion of this treatment is likely to have exacerbated disparities in health outcomes within hospitals.

In sum, we do not find evidence that black patients have sorted or migrated to higher-quality hospitals. These results are therefore supportive of models in which there is little convergence in mortality across hospitals between black and white AMI patients (Skinner and Staiger 2015). To the extent that more productive hospitals with better outcomes expand by increasing volume (as in Chandra et al. 2013), they may do so by drawing from people with similar race or ethnicities, and less through a reduction in segregation of hospital admissions.

\subsection{Literature and Theory}

The standard model of racial disparities in health care focuses on the clinical encounter, and embodies the idea that a provider treats two identical patients, one white and the other black, differently. Treatment differences in the clinical encounter may occur because there is explicit discrimination where a provider consciously withholds valuable care from minority patients. But disparities may also arise from implicit discrimination, where a harried provider operating in a time-sensitive environment makes unconscious mental decisions that are detrimental to minorities. Stereotyping is one manifestation of this indiscretion and it occurs when a provider uses a patient's race to deduce information about the benefit of treatment (Balsa and McGuire 2003). If African American patients are perceived as less likely to be compliant, then a physician may assume that her African American patient is less compliant. Such biases are compounded by poor communication between providers and their patients, which may create enormous psychological barriers to minority patients seeking care (van Ryn and Burke 2000). Similarly, minority patients may be treated by different physicians within a hospital, for example, by residents rather than attending physicians. If the quality of care differs between black and white patients, we would term this "within-hospital" disparities. ${ }^{1}$

Our earlier work focused on a different source of racial disparities capturing the idea that racial disparities in care are partially the consequence of differences in where minorities and whites receive care. Using five years of Medicare data from 1997 to 2001, Skinner et al. (2005) found that racial

1. Strumpf (2011) found that variations in quality of primary care by physicians varied more with respect to the specific physician providing the care than it did with regard to the match, or "concordance," of patient race or ethnicity and the physician's race or ethnicity. To the extent that physician quality varies across hospitals, this would again argue for between-hospital disparities rather than within-hospital disparities. 
disparities could primarily be attributed to the treatment of black patients at hospitals with poor risk-adjusted outcomes rather than by worse outcomes for black patients within hospitals. This finding was consistent with studies indicating that black patients are more likely to be treated at hospitals with lower rates of evidence-based treatments and protocols and higher surgical mortality (Barnato et al. 2005; Bradley et al. 2004; Konety, Vaughan Sarrazin, and Rosenthal 2005; Rothenberg et al. 2004).

Differences in where minorities are treated have to do with factors such as insurance and lower socioeconomic status, as well as historical patterns of discrimination and neighborhood segregation. Yet geographical treatment patterns, even for AMI patients being rushed to the hospital, are not set in stone. As Doyle et al. (2015) have found, hospital treatment patterns for AMI patients are strongly affected by which ambulance firm picks up the patient, so the potential is great for regionalization policies to encourage ambulances (and patients) to seek care at high-volume cardiac centers rather than low-volume or low-quality hospitals.

The empirical question is whether there should be convergence in quality across hospitals. If the reason why hospitals exhibit lower quality is because they are less likely to adopt new and effective technologies, or because they are more likely to adopt low-quality technologies, as in Skinner and Staiger (2015), then we might not expect convergence. As the lagging hospitals finally adopt the innovations that rapid-adopting hospitals have been using for years, the rapid-adoption hospitals have already moved on to a new technology, leading to a lack of convergence. Indeed, Skinner and Staiger (2015) found no evidence of convergence, in the sense that the lagging hospitals with low diffusion rates caught up to the rapid-adoption hospitals with high diffusion rates.

Conversely, more productive hospitals, in the sense of providing better health outcomes for AMI patients, have been shown to experience more rapid growth in patient populations (Chandra et al. 2013). In their model, demand-side factors would lead to convergence in hospital quality over time. A variant of this hypothesis would be that more productive hospitals would draw patients of similar race and ethnicity, but not draw new patients from across race and ethnicity; in this case we could observe a convergence in hospital quality within white and black patient populations, but no convergence between racial or ethnic groups.

Our analytic framework is a simple Oaxaca-Blinder decomposition exercise. At a point in time, the black-white difference in the probability of person $i$, of race $j$ and in year $t$, dying post-AMI, $m_{j t i}$, is given by

$$
m_{j t i}=x_{j t i} \beta_{j}+\delta_{k t}+\epsilon_{j t i} .
$$

In this linear probability model, mortality is a function of an individual's risk characteristics $x_{j t i}$, times the race-specific coefficient $\beta_{j}$, plus the hospitaland time-specific quality measure $\delta_{k t}, k=1, \ldots K$. The hospital-specific ef- 
fect captures a variety of different treatment approaches (and expertise), as well as differences in treatment of patients within hospitals by race. For example, if white AMI patients are more likely to receive primary reperfusion than black patients in hospital $k$, then $\delta_{k w}$ will be more likely to be much less than $\delta_{k b}$ (that is, a lower mortality rate for white patients in that hospital than for black patients in the same hospital).

The average mortality between black and white AMI patients, again at a point in time is

$$
M_{b t}-M_{w t}=\left(X_{b t}-X_{w t}\right) \beta+\sum_{k=1}^{K}\left[\delta_{k}\left(\mu_{b k}-\mu_{w k}\right)\right]+\omega,
$$

where $M_{k t}$ is the average mortality, $X$ the average value for the vector of comorbidities and risk adjusters, $Z$ the average value of the treatments within hospitals, $\mu_{j k}$ is the mean fraction of people of race or ethnicity $j$ who are admitted to hospital $j$, and $\omega_{t}$ captures the additional terms in general OaxacaBlinder decompositions reflecting differences in black and white coefficients (since we now assume a common $\beta$ for black and white patients), and thus reflects a residual term. A simplified version of the hospital-specific effect of racial representation creates 10 deciles of hospitals where hospitals are allocated into deciles depending on the fraction of black patients admitted for AMI.

\subsection{Data and Methods}

The data set used was a 100 percent sample of Medicare Provider Analysis and Review (MEDPAR) data on Medicare fee-for-service billing information for all patients hospitalized for AMI between January 1993 and December 2010, with follow-up data extending to December 2011. The sample was further restricted to only patients seen at hospitals that treated at least ten Medicare patients for AMIs. The criterion for determining the presence of AMI from the claims was a hospital admission with a primary diagnosis of AMI, without evidence of an AMI in the prior year.

Using information from the Medicare Denominator File, patients were categorized as black and white, ${ }^{2}$ while other race/ethnicity groups were excluded because of small sample sizes. The initial sample comprised 4,250,422 qualifying AMI events. Of these, 149,380 (3.5 percent) patients were excluded because they were neither black nor white, and an additional 3,556 ( 0.08 percent $)$ patients were excluded because they were seen at hospitals that treated fewer than ten patients for AMI in the study period. This left 4,097,486 (96 percent of original sample) patients who were assigned to the first hospital recording an AMI primary diagnosis code, regardless of future treatment location.

2. There is a very strong correlation between black racial measures in the Medicare claims data and self-reported racial identity (Arday et al. 2000). 
This sample was used to calculate the percentage of all AMI patients in a hospital who were black. We then created approximate deciles of this measure to provide a summary measure of the extent to which a hospital serves the black community. Patient counts in each of these deciles were not exactly 10 percent of the sample because patients in a given hospital were retained in the same decile category. We report summary statistics for each decile in table 6.1 .

The primary measure of outcomes was risk-adjusted mortality at ninety days from initial admission (sensitivity analysis is described below). Ninetyday mortality is likely to capture the total outcome of decisions that affect both short- and medium-term mortality (such as coronary artery bypass graft surgery, which can reduce medium-term mortality, but carries shortterm perioperative risk), while still capturing differences in hospital care, rather than differences in postacute care. Previous uses of these outcome data have been described elsewhere (McClellan, McNeil, and Newhouse 1994; McClellan and Noguchi 1998; Kessler and McClellan 2000; Skinner et al. 2003). As noted by previous studies, measures of hospital performance that use patient outcome data can be biased if hospitals serve patients with different average severity levels, however, measures of risk-adjusted AMI

Table 6.1

Distribution of patients and hospitals across years within study sample

\begin{tabular}{lccc}
\hline Year & Number of black patients & Total patients & Number of hospitals \\
\hline 1993 & 14,148 & 239,345 & 4,864 \\
1994 & 14,833 & 242,804 & 4,861 \\
1995 & 14,911 & 244,791 & 4,837 \\
1996 & 15,139 & 244,264 & 4,788 \\
1997 & 15,162 & 238,913 & 4,722 \\
1998 & 15,350 & 239,590 & 4,705 \\
1999 & 16,335 & 243,939 & 4,669 \\
2000 & 16,990 & 249,819 & 4,662 \\
2001 & 17,609 & 250,342 & 4,643 \\
2002 & 18,268 & 255,005 & 4,553 \\
2003 & 18,516 & 252,374 & 4,471 \\
2004 & 17,542 & 239,676 & 4,497 \\
2005 & 16,157 & 223,321 & 4,414 \\
2006 & 15,124 & 204,796 & 4,253 \\
2007 & 13,772 & 192,606 & 4,185 \\
2008 & 13,657 & 188,034 & 4,154 \\
2009 & 13,103 & 175,142 & 4,077 \\
2010 & 12,962 & 172,636 & 4,037 \\
Total & 279,578 & $4,097,397$ & 6,250 \\
\hline
\end{tabular}

Notes: The sample used is a census of the Medicare fee-for-service population hospitalized for AMI between January 1993 and December 2010. The sample was further restricted to only patients seen at hospitals that treated at least ten Medicare patients for AMIs. The criterion for determining the presence of AMI from the claims was a hospital admission with a primary diagnosis of AMI, without evidence of an AMI within the prior year. 
mortality have been shown to be valid indicators of hospital quality and have been incorporated into hospital profiling efforts (Davies et al. 2001).

This method of risk adjustment is not intended to be a comprehensive measure of a patient's well-being, as it leaves out a wide variety of relevant information, ranging from tobacco smoking status to clinical impressions and lab values. This set of controls is not sufficiently comprehensive to eliminate omitted variable bias, but it gives insight into the direction of that bias. As long as the unobserved health status of a patient is correlated with their observed health status, we can identify the direction of the bias, but not the magnitude.

To adjust for risk, we control for age nonparametrically in five-year increments, and allow each age category to interact with sex. We control for preexisting conditions using indicator variables for vascular disease, pulmonary disease, dementia, diabetes, renal failure, and cancer. Also included were year categorical variables and categorical variables that indicate the severity of the AMI, whether anterior, inferior, subendocardial, or a reference "other" category.

There are a range of potential health outcomes that are of interest following an AMI, including diminished cardiac output, reduced cognitive function and loss of independence, but we use mortality because it is unambiguous and reported with high reliability. While it would be of interest to study the incidence of congestive heart failure (CHF) following an AMI, the reporting and diagnosis of $\mathrm{CHF}$ depends on both the judgment of treating physicians, as well as the patient's access to follow-up care.

We estimated logistic regression models for both mortality and PCI use by first calculating year-specific rates by race, or aggregating the data to create early (1993-1998), middle (1999-2005), and late (2006-2010) rates of mortality, again by race. For ninety-day mortality, this is the basic racial disparity we seek to explain (that is, the left-hand side of equation 1). We then add comorbidities (thus capturing the effect of average differences in patient characteristics $X$ ) and hospital effects (capturing the average differences in hospital quality).

A simple way to demonstrate the "across-hospital" effect is to calculate the mortality rate for white patients if they had been admitted to hospitals in the same proportion as for black patients. In other words, if 1.4 percent of all black AMI patients were admitted to Hospital $\mathrm{H}$, and the white mortality rate there was 19.2 percent, then the weighted average of this "white patients admitted to black hospitals" measure would reflect the 19.2 percent white mortality rate but weighted by the percentage of black patients, 1.4 percent (see Skinner et al. 2005).

All statistical analysis was performed in STATA 13.1. 


\subsection{Results}

Table 6.1 presents the distribution of patients and hospitals across years within study sample. There is a rise in the number of AMI patients initially, reflecting a growth in the Medicare population, but the total number then declines, particularly near the end of the period, for two reasons. First, there was a rise in patients moving into Medicare Advantage managed care, and thus out of fee-for-service care, leading to a smaller denominator of feefor-service patients over time. And second, there has been a general decline in the incidences of AMI (Likosky et al. 2013).

Table 6.2 presents summary statistics for fee-for-service Medicare beneficiaries who were treated for AMI between 1993 and 2010. The table illustrates the construction of the deciles used in the analysis, as well as the wide variation in the racial composition of patients at different hospitals. Focusing on the statistics at the bottom of the left column, the average Medicare AMI patient was treated in a hospital where 6.82 percent of AMI patients were black. The typical black patient, however, was treated at a hospital where 23.57 percent of all patients were black, while the typical white patient was treated at a hospital where just 5.60 percent of patients were black. Patients treated at hospitals with the highest proportion of black patients were disproportionately from the South, and living in poorer ZIP codes (not reported).

In figure 6.1, panels A, B, and C, we show the fraction of black and white AMI patients by decile for each of the three periods: 1993-1998, 19992005, and 2006-2010. It is clear that black and white AMI patients tend to be admitted to different hospitals (as in Skinner et al. 2005). However, there does not appear to be a trend in the extent to which black and white patients are admitted to largely different hospitals. Thus, as a first test, there is no evidence of convergence in the degree of segregation of hospital AMI admissions.

One concern is that white patients in disproportionately black hospitals are in worse health to begin with; thus, it would be no surprise to show that such patients experience worse health outcomes owing to unmeasured comorbidities. Figure 6.2 presents an index of AMI severity across each of the ten hospital deciles; this measures predicted mortality based on recorded comorbidities and AMI presentation, but controls for age, race, sex, and the racial composition of the patients treated at the same hospital. There do not appear to be differences in the underlying risk of mortality across hospital type, making it less likely that unobservable confounding variables could explain the differences we find (Altonji et al. 2010).

Figure 6.3 shows regression estimates from a model that risk adjusts and allows for different mortality rates by race and by hospital decile. While the confidence intervals are fairly wide, they still demonstrate that for the time 


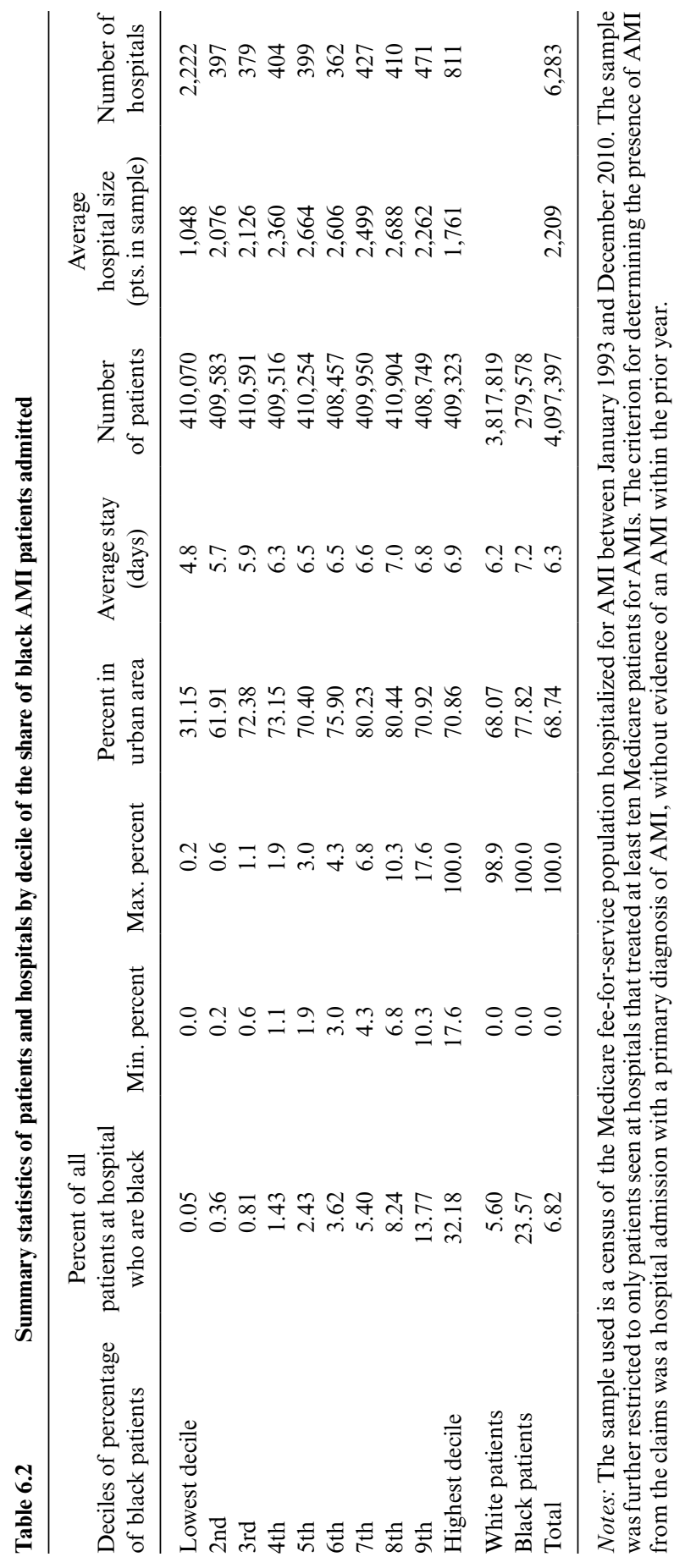



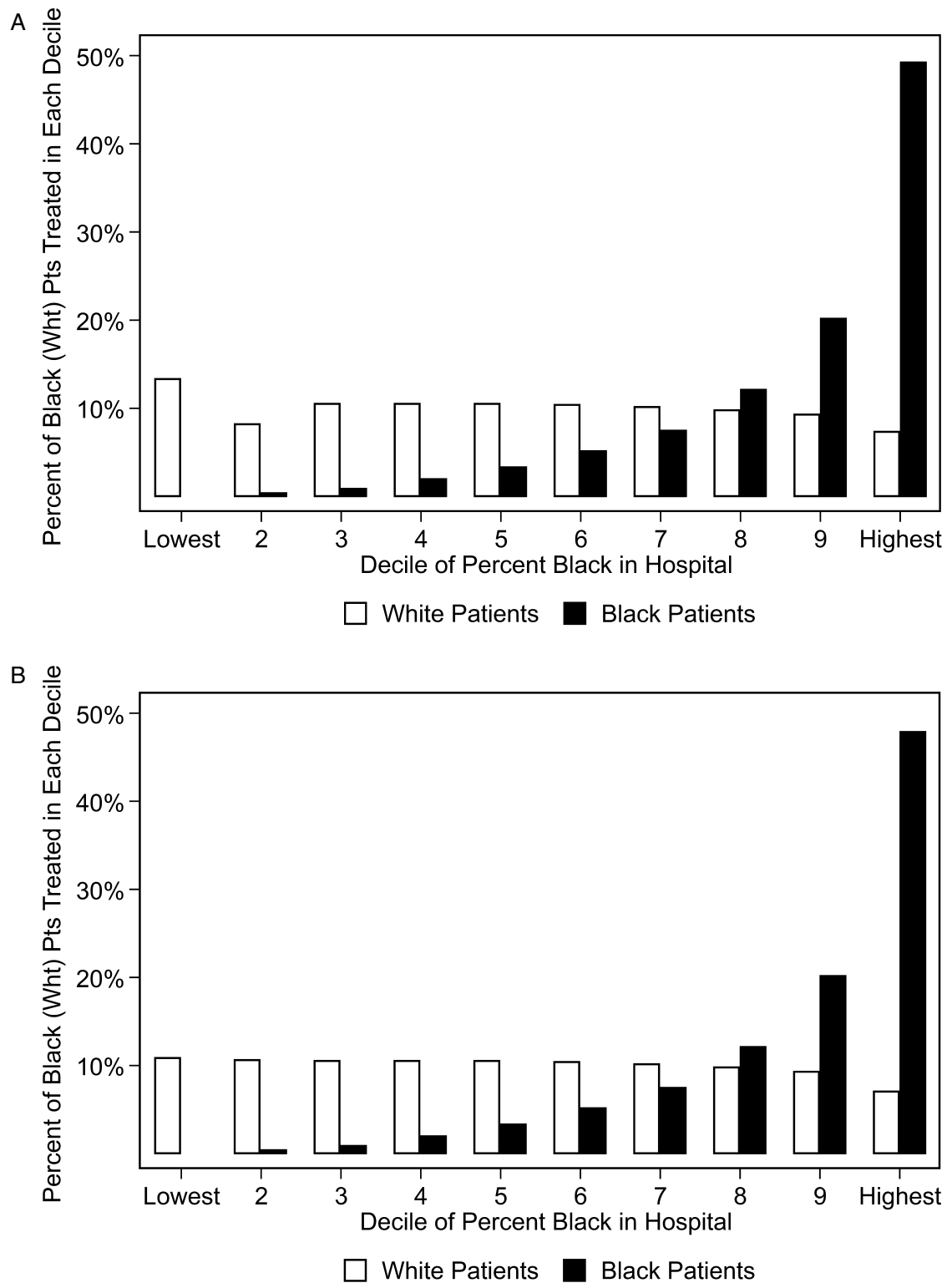

Fig. 6.1 Distribution of black and white patients by percent black admitted to hospital

Notes: Panel A: Distribution of patients by race (1993-1998); panel B: Distribution of patients by race (1999-2005). After discarding hospitals with fewer than ten patients over the study period, hospitals are ranked by the degree to which they serve the black population over the entire study period. The bars titled "Black Patients" indicate the percent of the black population that is treated in each grouping of hospitals. 


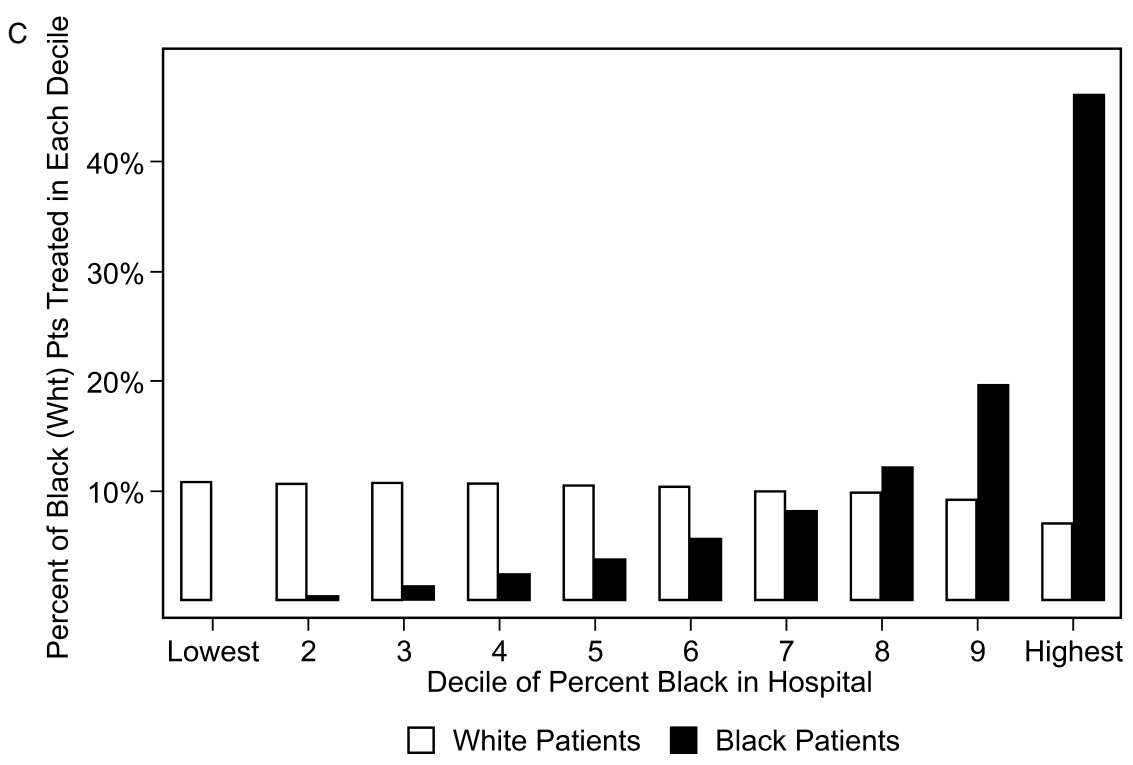

Fig. 6.1 (cont.)l

Note: Panel C: Distribution of patients by race (2006-2010).

period we consider, most of the variation in mortality arose from the hospital to which patients were admitted, rather than differences that occurred within the hospital. (Figure 6.4 shows the same results, but with Hospital Referral Region [HRR] fixed effects).

Figure 6.5 shows time trends in the risk-adjusted ninety-day mortality risk. For both black and white patients there was a marked decline in mortality during this period, but there is no evidence of less overall disparity in hospital mortality. As detailed in table 6.3, during the later period (2006-2010) there was a gap of 1 percentage point between black ninetyday mortality (18.8 percent) and white ninety-day mortality (17.8 percent). Had the white patients been admitted to hospitals using the same frequency as black patients, then the average white mortality rate would have been 18.5 percent. The within-hospital racial disparity is therefore 0.3 percent (18.8 percent-18.5 percent) and the across-hospital disparity is the remaining 0.7 percent, a slight reduction from the 0.9 percent gap in the early period and the 1.0 percent difference in the middle period.

The introduction of HRR fixed effects does not substantially change the pattern of results that we find, suggesting that the broad patterns of between-hospital disparity we find are not driven by regional differences. In other words, the evidence is weak that patient migration (or shutting down of hospitals) has led to a reduction in disparities across hospitals. 
Comorbidity Index based on 90-day Mortality (White Patients)

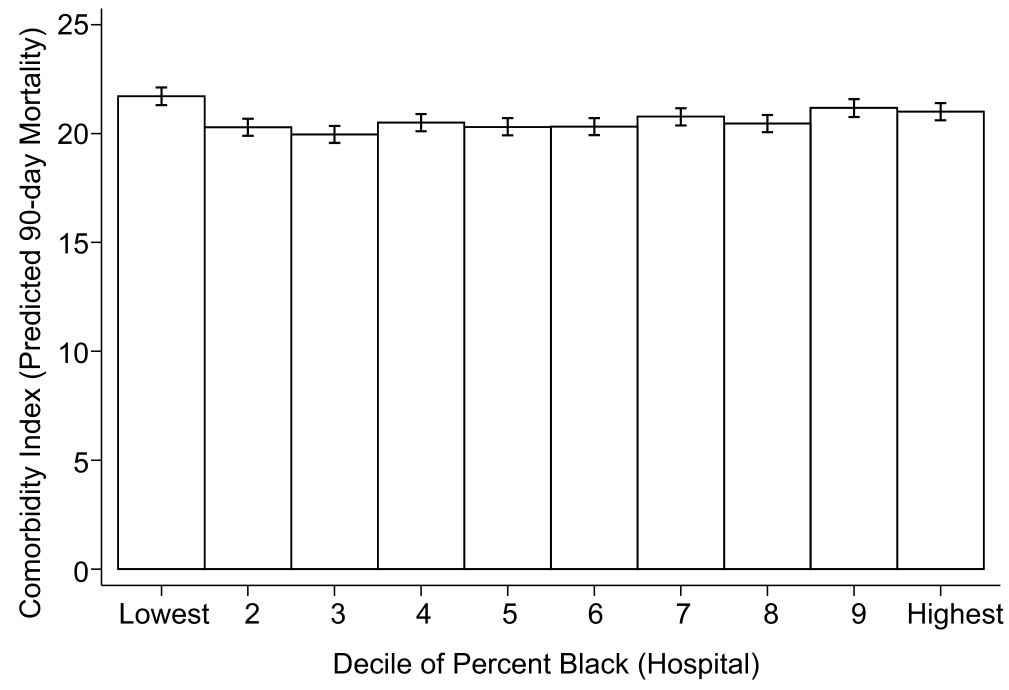

Fig. 6.2 Index of comorbidity and AMI severity by percent of admitted patients who are black

Note: Figure reports predicted ninety-day mortality for white patients across deciles of percent black. Predicted mortality is calculated using all the risk adjusters in the data, including age and sex. Risk adjustment includes controls for vascular disease, pulmonary disease, dementia, diabetes, renal failure, cancer, and the location of the heart attack within the heart: anterior, inferior, subendocardial, or a reference "other" category.

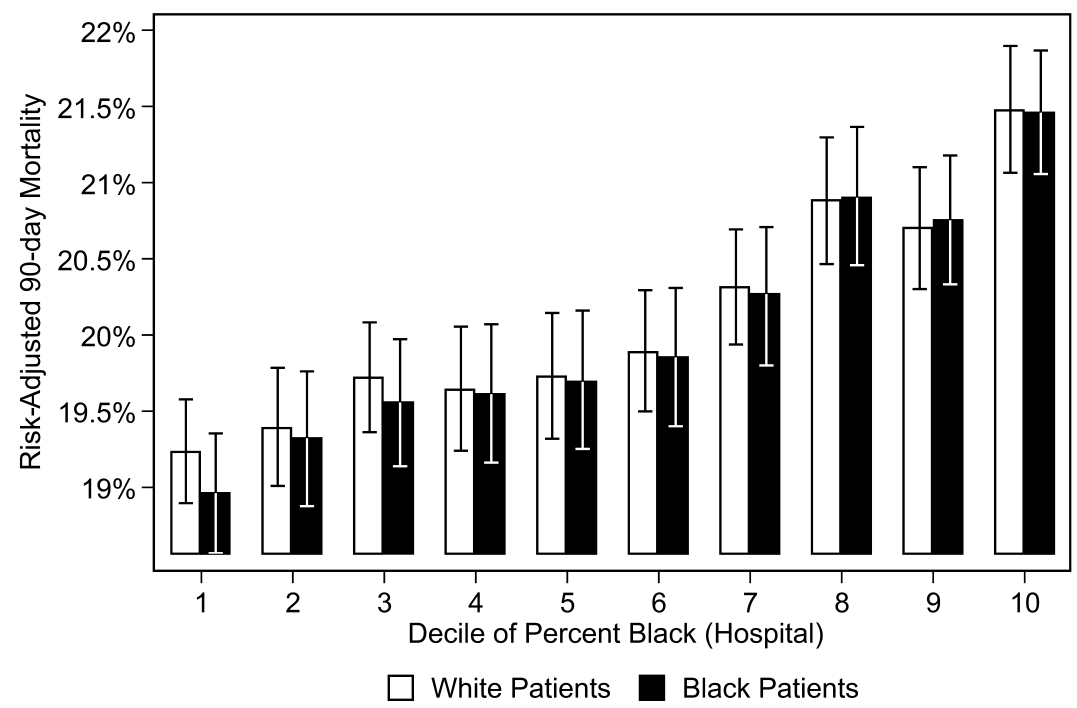

Fig. 6.3 Risk-adjusted mortality by hospital decile and race 


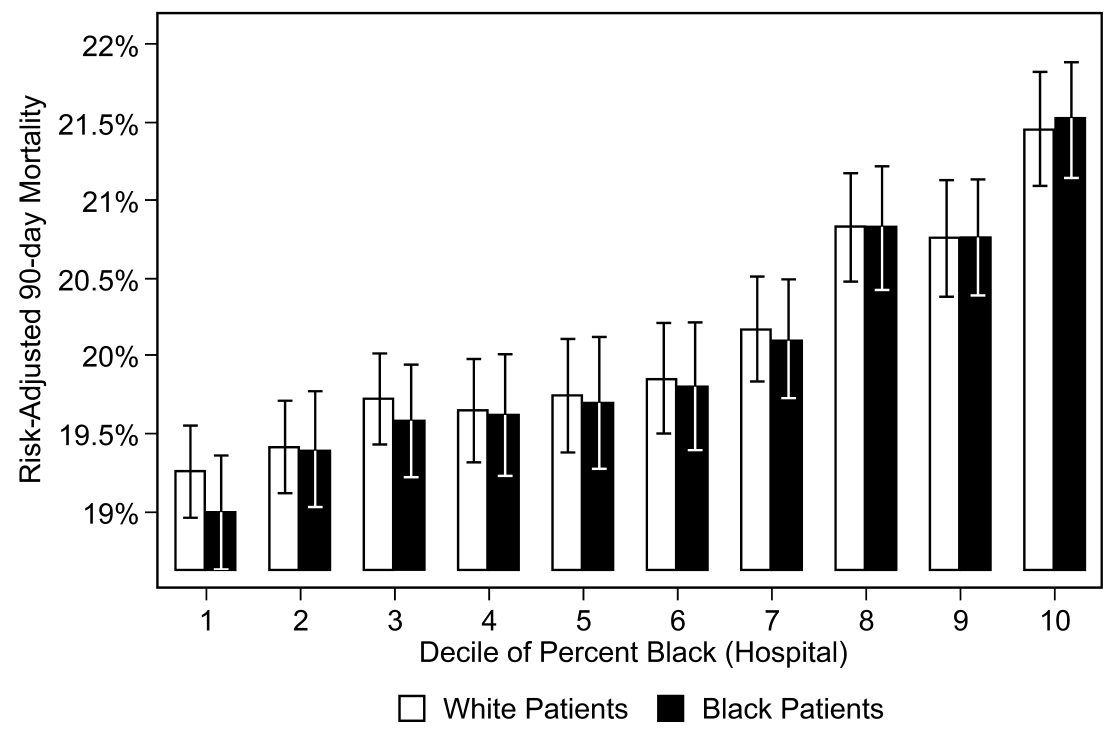

Fig. 6.4 Risk- and HRR-adjusted mortality by hospital decile and race

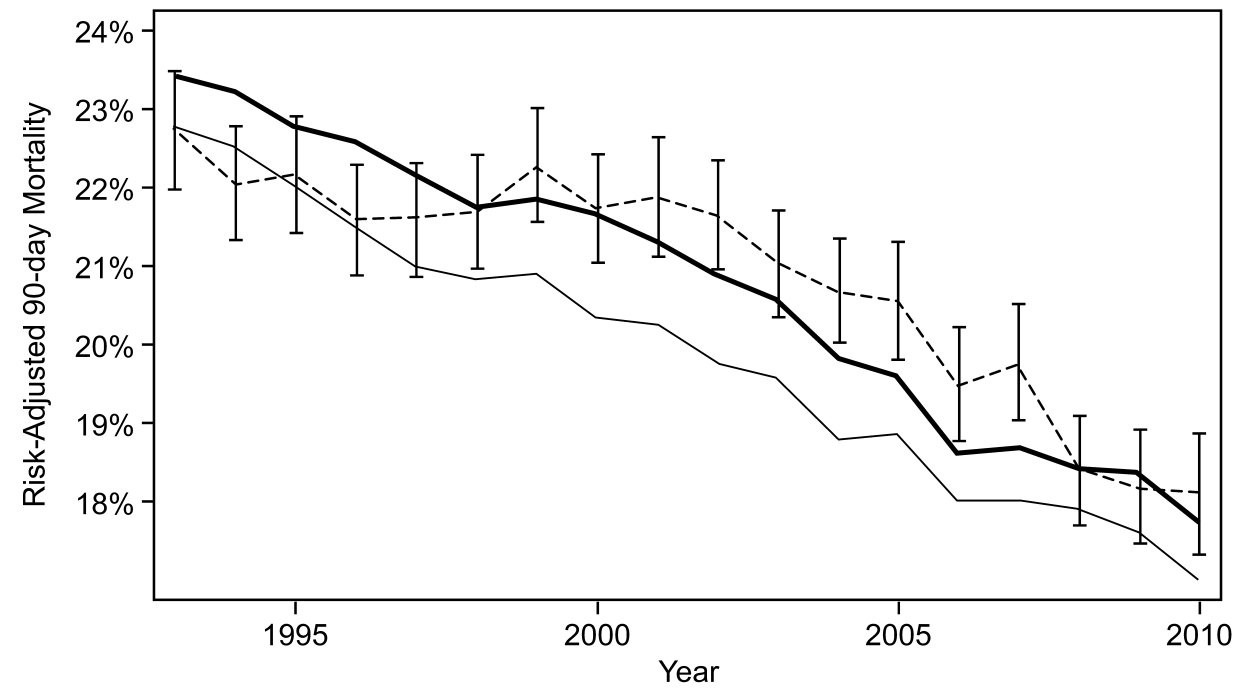

White Patients Treated at Black Hospitals ---- Black Patients White Patients

Fig. 6.5 Risk-adjusted ninety-day AMI mortality

Note: Risk adjustment includes controls for age, sex, vascular disease, pulmonary disease, dementia, diabetes, renal failure, cancer, and the location of the heart attack within the heart: anterior, inferior, subendocardial, or a reference "other" category. 
Table 6.3

Mortality rates at thirty-day, ninety-day, and one-year horizons by race and hospital admission patterns

\begin{tabular}{|c|c|c|c|c|}
\hline & $\begin{array}{c}\text { Overall } \\
(\%)\end{array}$ & $\begin{array}{c}\text { Early } \\
(1993-1998) \\
(\%)\end{array}$ & $\begin{array}{c}\text { Middle } \\
(1999-2005) \\
(\%)\end{array}$ & $\begin{array}{c}\text { Late } \\
(2006-2010) \\
(\%)\end{array}$ \\
\hline \multicolumn{5}{|c|}{ Ninety days } \\
\hline White & 19.7 & 21.7 & 19.8 & 17.8 \\
\hline White (unadjusted) & 19.7 & 23.1 & 20.3 & 17.8 \\
\hline Black & 20.7 & 22.1 & 21.4 & 18.8 \\
\hline Black (unadjusted) & 20.8 & 24.1 & 23.0 & 20.1 \\
\hline White, admitted to black hospitals & 20.6 & 22.6 & 20.8 & 18.5 \\
\hline $\begin{array}{l}\text { White, admitted to black hospitals } \\
\text { (unadjusted) }\end{array}$ & 20.7 & 24.2 & 21.3 & 18.5 \\
\hline \multicolumn{5}{|c|}{ Thirty days } \\
\hline White & 14.6 & 16.4 & 14.6 & 13.1 \\
\hline White (unadjusted) & 14.6 & 18.3 & 15.2 & 12.8 \\
\hline Black & 14.6 & 15.7 & 15.0 & 13.1 \\
\hline Black (unadjusted) & 14.6 & 18.0 & 16.2 & 13.3 \\
\hline White, admitted to black hospitals & 15.3 & 17.0 & 15.3 & 13.6 \\
\hline $\begin{array}{l}\text { White, admitted to black hospitals } \\
\text { (unadjusted) }\end{array}$ & 15.3 & 19.2 & 15.9 & 13.3 \\
\hline \multicolumn{5}{|c|}{ One year } \\
\hline White & 28.6 & 31.2 & 28.8 & 25.9 \\
\hline White (unadjusted) & 28.6 & 31.4 & 29.4 & 26.9 \\
\hline Black & 32.0 & 33.5 & 32.8 & 29.6 \\
\hline Black (unadjusted) & 32.0 & 35.5 & 35.7 & 33.2 \\
\hline White, admitted to black hospitals & 29.7 & 32.4 & 30.0 & 26.6 \\
\hline $\begin{array}{l}\text { White, admitted to black hospitals } \\
\text { (unadjusted) }\end{array}$ & 29.7 & 32.8 & 30.8 & 27.9 \\
\hline
\end{tabular}

Note: The sample used is a census of the Medicare fee-for-service population hospitalized for AMI between January 1993 and December 2010. The sample was further restricted to only patients seen at hospitals that treated at least ten Medicare patients for AMIs. The criterion for determining the presence of AMI from the claims was a hospital admission with a primary diagnosis of AMI, without evidence of an AMI within the prior year. Risk adjustment includes controls for vascular disease, pulmonary disease, dementia, diabetes, renal failure, cancer, and the location of the heart attack within the heart: anterior, inferior, subendocardial, or a reference "other" category.

Nor is there evidence of a decline in within-hospital disparities during this period - the "black" versus "white admitted to black hospital" lines are nearly parallel.

We also consider alternative mortality horizons, shown in table 6.3 with one-year, thirty-day, and ninety-day mortality rates for three groups: black AMI patients, white AMI patients, and white AMI patient mortality weighted by hospital admission patterns for black AMI patients, across the three periods of analysis. There is a clear gradient showing worse out- 
comes for black AMI patients at longer horizons. While the gap in betweenhospital mortality variation is larger at 365 days ( 1.2 percent in the early period, and 1.3 percent in the middle period), there is a modest decline as for the later period ( 0.8 percent).

Why are health outcomes so much worse for black patients at longer horizons? One reason might be that we have not adequately adjusted for other comorbidities that affect longer-term longevity. In a separate analysis (not reported here), we considered this puzzle using more detailed risk adjustment based on HCC scores (hierarchical condition category) for more recent data. While HCC scores are known to be sensitive to overadjustment (e.g., Einav et al. 2015; Song et al. 2010), our results suggested that much of the gap at both thirty days and 365 days could be explained by differential adjustment. Thus, we are more confident about changes over time in black and white rates than about comparing black and white rates at a point in time.

Why might there be so little gain in within-hospital mortality disparity? In the early period, the within-hospital gap was -0.5 percent (that is, white mortality rates, with hospital weights determined by black hospital admissions, were higher than for black mortality rates with the same hospital

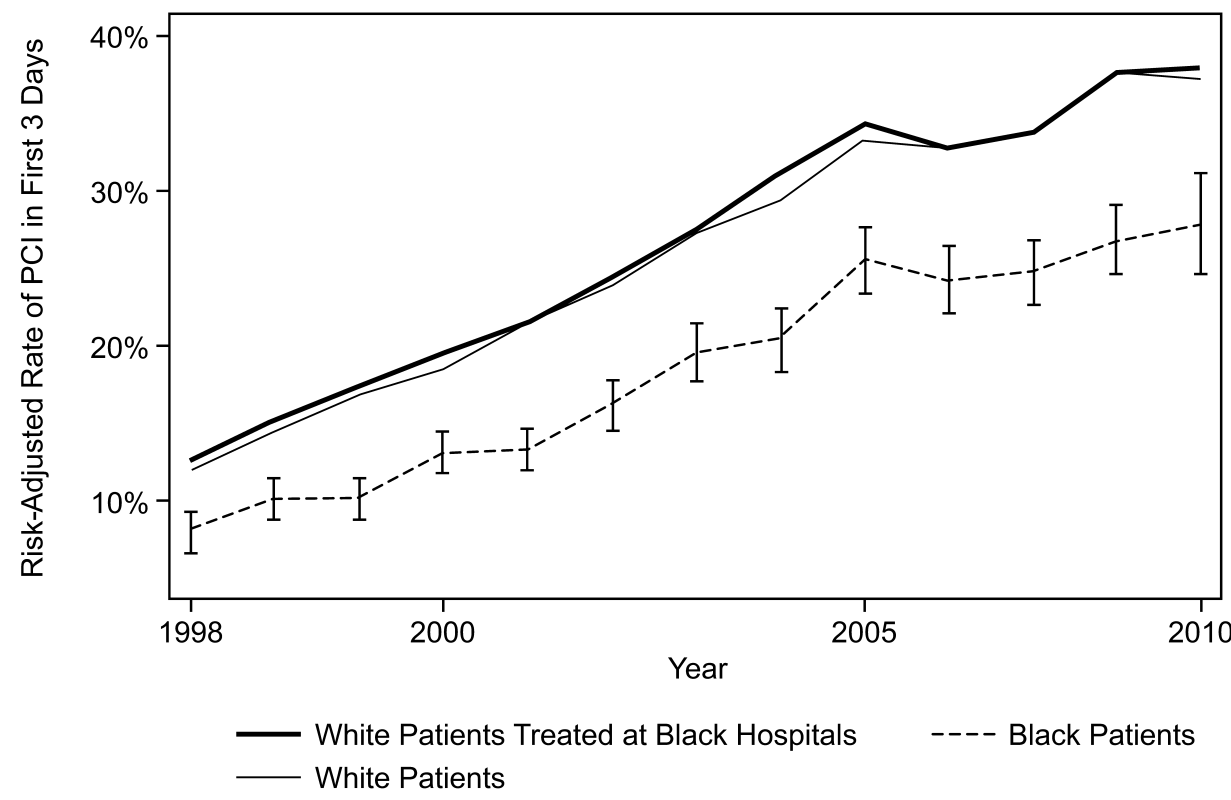

Fig. 6.6 Risk-adjusted rate of prompt PCI

Note: Rates of PCI in the first three days after hospital admission have climbed dramatically, with black patients receiving PCI at rates significantly below whites. This gap persists through the study period, and does not close in the later years. 
weights). In the later period, nearly two decades later, the within-hospital gap had flipped to 0.7 percent. One reason is shown in figure 6.6; rates of percutaneous coronary interventions (PCI), which have been steadily rising for all patients over this period, but where the absolute gap in PCI rates has been increasing even more rapidly. While the proportional difference may have shrunk during this period, what matters for the gap in mortality is the incremental value of the procedure times the fraction of AMI patients who receive the treatment. And this has been rising, from a few percentage points in the mid-1990s, to roughly 10 percentage points in 2010 .

\subsection{Conclusion}

During the 1993-2010 period, there was a tremendous increase in the scientific literature on racial disparities in health care. Based on these findings, one would have expected proactive efforts by providers to narrow health disparities, particularly for disparities occurring within hospitals. For acute myocardial infarction in the elderly population, we observe a remarkable decline in mortality over the past several decades. However, the decline in mortality was not associated with a reduction in mortality differentials within hospitals, nor did we observe a significant reduction in mortality disparities associated with black AMI patients being admitted to hospitals with disproportionately high risk-adjusted mortality rates for whites. While there was some hint of an improvement in the across-hospital gap between the middle (1999-2005) and late (2006-10) periods, additional years of data would be necessary to discern whether it was statistical noise or a long-term improvement.

The fact that black and white AMI patients go to different hospitals is, in many respects, the consequence of racial segregation in where people live, and not a systematic effort to discriminate against black AMI patients. That is, we should not be surprised to find that AMI patients living in Mississippi are more likely to be admitted to hospitals with disproportionately black patient populations than AMI patients living in North Dakota. Of greater interest for policy purposes is segmentation of markets not driven by distance alone, that is, black patients who are admitted to lower-quality hospitals when there are higher-quality hospitals nearby. Studying these more granular travel patterns is a topic for future research.

\section{References}

Altonji, Joseph G., Timothy Conley, Todd E. Elder, and Christopher R. Taber. 2010. "Methods for Using Selection on Observed Variables to Address Selection on Unobserved Variables." Unpublished Manuscript. 
Arday, S. L., D. R. Arday, Stephanie Monroe, and J. Zhang. 2000. "HCFA's Racial and Ethnic Data: Current Accuracy and Recent Improvements." Health Care Financing Review 21 (4): 107-16.

Balsa, Ana I., and Thomas G. McGuire. 2003. "Prejudice, Clinical Uncertainty and Sterotyping as Sources of Health Disparities." Journal of Health Economics 22 (1): 89-116.

Barnato, Amber E., F. Lee Lucas, Douglas Staiger, David E. Wennberg, and Amitabh Chandra. 2005. "Hospital-Level Racial Disparities in Acute Myocardial Infarction Treatment and Outcomes." Medical Care 43 (4): 308-19.

Bradley, Elizabeth H., Jeph Herrin, Yongfei Wang, Robert L. McNamara, Tashonna R. Webster, David J. Magid, Martha Blaney, et al. 2004. "Racial and Ethnic Differences in Time to Acute Reperfusion Therapy for Patients Hospitalized with Myocardial Infarction." Journal of the American Medical Association 292 (13): 1563-72.

Chandra, Amitabh, Amy Finkelstein, Adam Sacarny, and Chad Syverson. 2013. "Healthcare Exceptionalism? Productivity and Allocation in the US Healthcare Sector.” NBER Working Paper no. 19200, Cambridge, MA.

Davies, Sheryl M., Jeffrey Geppert, Mark McClellan, Kathryn M. McDonald, Patrick S. Romano, and Kaveh G. Shojania. 2001. "Refinement of the HCUP Quality Indicators.” Agency for Healthcare Research and Quality (US). http://archive .ahrq.gov/clinic/tp/hcupqitp.htm.

Doyle, Joseph, John Graves, Jonathan Gruber, and Samuel Kleiner. 2015. Measuring Returns to Hospital Care: Evidence from Ambulance Referral Patterns." Journal of Political Economy 123 (1): 170.

Einav, Liran, Amy Finkelstein, Raymond Kluender, and Paul Schrimpf. 2015. "Beyond Statistics: The Economic Content of Risk Scores." NBER Working Paper no. 21304, Cambridge, MA.

Institute of Medicine (IOM). 2002. Unequal Treatment: Confronting Racial and Ethnic Disparities in Health Care. Washington, DC: National Academy Press.

Jha, A., E. S. Fisher, Z. Li, E. J. Orav, and A. M. Epstein. 2005. "Racial Trends in the Use of Major Procedures among the Elderly." New England Journal of Medicine 353 (7): 683-91.

Kessler, Daniel P., and Mark B. McClellan. 2000. "Is Hospital Competition Socially Wasteful?" Quarterly Journal of Economics 115 (2): 577-615.

Konety, Suma H., Mary S. Vaughan Sarrazin, and Gary E. Rosenthal. 2005. "Patient and Hospital Differences Underlying Racial Variation in Outcomes after Coronary Artery Bypass Graft Surgery." Circulation 111:1210-16.

Likosky, Donald S., Weiping Zhou, David J. Malenka, William B. Borden, David J. Malenka, William B. Borden, Brahmajee K. Nallamothu, and Jonathan Skinner. 2013. "Growth in Medicare Expenditures for Patients with Acute Myocardial Infarction: A Comparison of 1998 through 1999 and 2008." JAMA Internal Medicine 173 (22): 2055-61.

McClellan, M., B. J. McNeil, and J. P. Newhouse. 1994. "Does More Intensive Treatment of Acute Myocardial Infarction in the Elderly Reduce Mortality? Analysis Using Instrumental Variables." Journal of the American Medical Association 272 (11): 859-66.

McClellan, M., and Haruko Noguchi. 1998. "Technological Change in HeartDisease Treatment. Does High Tech Mean Low Value?" American Economic Review (Proceedings) 88 (2): 90-96.

Meara, Ellen R., Seth Richards, and David M. Cutler. 2008. "The Gap Gets Bigger: Changes in Mortality and Life Expectancy, by Education, 1981-2000." Health Affairs 27 (2): 350-60. 
Rothenberg, Barbara M., Thomas Pearson, Jack Zwanziger, and Dana Mukamel. 2004. "Explaining Disparities in Access to High-Quality Cardiac Surgeons." Annals of Thoracic Surgery 78:18-24. doi:10.1016/j.athoracsur.2004.01.021.

Skinner, Jonathan, Amitabh Chandra, Douglas Staiger, Julie Lee, and Mark McClellan. 2005. "Mortality after Acute Myocardial Infarction in Hospitals That Disproportionately Treat Black Patients." Circulation 112 (17): 2634-41.

Skinner, Jonathan, and Douglas Staiger. 2015. "Technology Diffusion and Productivity Growth in Health Care." Review of Economics and Statistics 97 (5): 951-64.

Skinner, Jonathan, James N. Weinstein, Scott M. Sporer, and John E. Wennberg. 2003. "Racial, Ethnic, and Geographic Disparities in Rates of Knee Arthroplasty among Medicare Patients." New England Journal of Medicine 349 (14): 1350-59.

Song, Yunjie, Jonathan Skinner, Julie Bynum, Jason Sutherland, John E. Wennberg, and Elliott S. Fisher. "Regional Variations in Diagnostic Practices." New England Journal of Medicine 363 (1): 45-53.

Strumpf, Erin C. 2011. "Racial/Ethnic Disparities in Primary Care: The Role of Physician-Patient Concordance." Medical Care 49 (5): 496-503.

Trivedi, Amal N., Regina C. Grebla, Steven M. Wright, and Donna L. Washington. 2011. "Despite Improved Quality of Care in the Veterans Affairs Health System, Racial Disparity Persists for Important Clinical Outcomes." Health Affairs 30 (4): 707-15.

Trivedi, Amal N., A. M. Zaslavsky, E. C. Schneider, and J. Z. Ayanian. 2005. "Trends in the Quality of Care and Racial Disparities in Medicare Managed Care." New England Journal of Medicine 353 (7): 692-700.

van Ryn, Michelle, and Jane Burke. 2000. "The Effect of Patient Race and SocioEconomic Status on Physician's Perceptions of Patients." Social Science and Medicine 50 (6): 813-28.

\section{Comment David R. Weir}

This chapter is a good example of the strengths of this research program in the economics of aging. It deals with a problem of first-order importance for the health and well-being of the older population. It utilizes data that are both appropriate and all-but-definitive for the question. The methods are careful but not overwrought, letting the data speak for themselves. The conclusion is well supported by the evidence, and points clearly to the next steps to be taken in the research agenda.

Racial disparities in health and aging are substantial. According to the National Center for Health Statistics (NCHS) life tables for 2010, life expectancy at age fifty is 3.3 years longer for white men than African Americans (12 percent longer), and 2.2 years longer for white women than for black

David R. Weir is a research affiliate of the Population Studies Center and a research professor at the Survey Research Center, both at the University of Michigan, and director of the Health and Retirement Study (HRS).

For acknowledgments, sources of research support, and disclosure of the author's material financial relationships, if any, please see http://www.nber.org/chapters/c13644.ack. 